\title{
New developments on the twin prime problem and generalizations
}

\author{
M. Ram Murty
}

\begin{abstract}
We give a short survey of some recent remarkable advances on the twin prime problem. Beginning with the breakthrough work of Zhang in May 2013, we trace the developments culminating in the work of Maynard and Tao in November 2013 that led to simplifications and numerical improvements of Zhang's work.
\end{abstract}

Keywords. sieve theory, twin primes, level of distribution

2010 Mathematics Subject Classification. 11N35, 11N36, 11N05

The year 2013 saw two major breakthroughs regarding the twin prime problem in the field of analytic number theory. The first took place in April and the second in October and November. The first event was quite dramatic and caught the mathematical community by surprise. On April 17, 2013, a relatively unknown mathematician from the University of New Hampshire, Yitang Zhang, submitted a paper to the Annals of Mathematics. The paper claimed to prove that there are infinitely many pairs of distinct primes $(p, q)$ with $|p-q|<7 \times 10^{7}$. This was a major step towards the celebrated twin prime conjecture! A quick glance at the paper convinced the editors that this was not a submission from a crank. The paper was written with impeccable clarity and demonstrated a consummate understanding of the latest technical results in analytic number theory. Therefore, the editors promptly sent it to several experts for refereeing. The paper was accepted three weeks later.

Zhang's theorem is really a corollary of a more important contribution he made in sieve theory. He extended the level of distribution in the Bombieri-Vinogradov theorem beyond the traditional $1 / 2$-level with the added proviso that we require the moduli in the range to be $\eta$-smooth for some small $\eta$. This is a rather technical statement, and we will explain it below. Suffice it to say that this result forms the bulk of Zhang's paper and uses the full artilery of 20th century sieve theory as well as contributions from algebraic geometry that went in to certain estimates of exponential sums.

In October 2013, Maynard and Tao, independently devised another way to deduce the bounded gaps result which is much simpler to use and also dispenses with the non-trivial algebraic geometry that was a key component of Zhang's proof. Not only was the proof simpler, but they also obtained better numerical results. The latest record is a bound of 246 . That is, there are infinitely many prime pairs $(p, q)$ with $0<|p-q| \leq 246$.

In this article, which is an expanded and updated version of my earlier article [Ram13a], we will outline the proofs of these recent breakthrough theorems of Yitang Zhang [Zha13], Maynard [May13] and Tao [Tao13]. Even though this article is only an outline, it should help the serious student to study these papers in greater detail. We want to emphasize that though the Maynard-Tao result is numerically better with respect to bounded gaps, the improvement of the Bombieri-Vinogradov theorem beyond the traditional $1 / 2$ level for smooth moduli, is a major result of independent interest and significance. For our exposition, the rudimentary background in analytic number theory is readily obtained from [Ram08] and [Dav00]. This can be followed by a careful study of [GPY09] and the three papers [BFI86], [BFI87], [BFI89]. 


\section{Introduction and history}

Let $p_{1}, p_{2}, \ldots$ be the ascending sequence of prime numbers. The twin prime problem is the question if there are infinitely many pairs of primes $(p, q)$ with $|p-q|=2$. This problem is usually attributed to the ancient Greeks, but this is very much Greek mythology and there is no documentary evidence to support it. The first published reference to this question appears in 1849 due to Alphonse de Polignac who conjectured more generally that for any given even number $2 a$, there are infinitely many pairs of primes such that $|p-q|=2 a$. In a recent paper [Zha13] in the Annals of Mathematics, Yitang Zhang proved that there are infinitely many pairs of distinct primes $(p, q)$ with

$$
|p-q|<7 \times 10^{7} .
$$

The bound was later improved to 246 using methods of Maynard and Tao and a simpler approach.

Zhang's proof depends on major milestones of 20th century number theory and algebraic geometry. Thus, it is definitely a 21st century theorem! Undoubtedly, his paper opens the door for further improvements and it is our goal to discuss some of these below. By contrast, the Maynard-Tao theorem depends only on results of analytic number theory that were available as early as 1960 .

After de Polignac's conjecture, the first serious paper on the subject was due to Viggo Brun in 1915, who, after studying the Eratosthenes sieve, developed a new sieve, now called Brun's sieve, to study twin primes and related questions. He proved that

$$
\sum_{p: p+2 \text { prime }} \frac{1}{p}<\infty .
$$

By contrast, the sum of the reciprocals of the primes diverges and so, this result shows that (in some sense) if there are infinitely many twin primes, they are very sparse.

A few years later, in 1923, Hardy and Littlewood [HL23], made a more precise conjecture on the number of twin primes up to $x$. They predicted that this number is

$$
\sim 2 \prod_{p>2}\left(1-\frac{1}{(p-1)^{2}}\right) \frac{x}{\log ^{2} x}
$$

(see p. 371 of [HW08]). They used the circle method, originally discovered by Ramanujan and later developed by Hardy and Ramanujan in their research related to the partition function. After Ramanujan's untimely death, it was taken further by Hardy and Littlewood in their series of papers on Waring's problem. (The circle method is also called "the Hardy-Littlewood method" by some mathematicians.) In the third paper of this series, they realized the potential of the circle method to make precise conjectures regarding additive questions, such as the Goldbach conjecture and the twin prime problem.

An alternate heuristic approach to making reasonable conjectures regarding the distribution of prime $k$-tuples is to use the notion of Ramanujan expansions. These are "expansions" of arithmetical functions in terms of Ramanujan sums and using an orthogonality principle for such sums, one can derive identical conjectures. For more details, we refer the reader to the author's paper in this journal [Ram13b].

Based on heuristic reasoning, it is not difficult to see why such conjectures should be true. The prime number theorem tells us that the number of primes $\pi(x)$, up to $x$, is asymptotically $x / \log x$. Thus, the probability that a random number in $[1, x]$ is prime is $1 / \log x$ and so the probability that both $n$ and $n+2$ are prime is about $1 / \log ^{2} x$. The constant is a bit more delicate to conjecture and is best derived using the theory of Ramanujan-Fourier series expansion of the von Mangoldt function alluded to earlier. We will not discuss this here but refer the reader to [Ram13b] for further details. 


\section{The basic strategy of Zhang's proof}

Let $\theta(n)=\log n$ if $n$ is prime and zero otherwise. We will use the notation $n \sim x$ to mean that $x<n<2 x$. Now, suppose we can find a positive real-valued function $f$ such that for

$$
\begin{gathered}
S_{1}=\sum_{n \sim x} f(n), \\
S_{2}=\sum_{n \sim x}(\theta(n)+\theta(n+2)) f(n)
\end{gathered}
$$

we have

$$
S_{2}-(\log 3 x) S_{1}>0,
$$

for $x$ sufficiently large. Then we can deduce that there exists an $n$ such that $n$ and $n+2$ are both prime with $x<n<2 x$. Such a technique and a method to choose optimal functions $f$ goes back to the 1950's and is rooted in the Selberg sieve. See for example [Ram08] for a short introduction to the Selberg sieve.

The problem as posed above is intractable. So we generalize the problem and consider sets

$$
\mathcal{H}=\left\{h_{1}, h_{2}, \ldots, h_{k}\right\} .
$$

It is reasonable to expect (under suitable conditions) that there are infinitely many $n$ such that $n+h_{1}, n+h_{2}, \ldots n+h_{k}$ are all prime. This would be a form of generalized twin prime problem and was first enunciated in the paper by Hardy and Littlewood alluded to above. Clearly, we need to put some conditions on $\mathcal{H}$. Indeed, if for some prime $p$ the image of $\mathcal{H}(\bmod p)$ has size $p$, then all the residue classes are represented by $\mathcal{H}$ so that in the sequence,

$$
n+h_{1}, n+h_{2}, \ldots, n+h_{k}
$$

there will always be some element divisible by $p$ and it is unreasonable to expect that for infinitely many $n$ all of these numbers are prime numbers. So a necessary condition is that $\nu_{p}(\mathcal{H})=|\mathcal{H}|(\bmod$ $p)<p$ for every prime $p$. Under such a condition, the set is called admissible and we expect this to be the only local obstruction.

Zhang [Zha13] proves:

Theorem 1. Suppose that $\mathcal{H}$ is admissible with $k \geq 3.5 \times 10^{6}$. Then, there are infinitely many positive integers $n$ such that the set

$$
\left\{n+h_{1}, \ldots, n+h_{k}\right\}
$$

contains at least two primes. Consequently,

$$
\liminf _{n \rightarrow \infty}\left(p_{n+1}-p_{n}\right)<7 \times 10^{7}
$$

Zhang shows the second assertion follows from the first if we choose for $\mathcal{H}$ a set of $k_{0}=3.5 \times 10^{6}$ primes lying in the interval $\left[3.5 \times 10^{6}, 7 \times 10^{7}\right]$. This can be done since

$$
\pi\left(7 \times 10^{7}\right)-\pi\left(3.5 \times 10^{6}\right)>3.5 \times 10^{6}
$$

from known explicit upper and lower bounds for $\pi(x)$ due to Dusart [Dus98]. That such a set of primes is admissible is easily checked. Indeed, if $p>k_{0}, \nu_{p}(\mathcal{H}) \leq k_{0}<p$. If $p \leq k_{0}$ and $\nu_{p}(\mathcal{H})=p$, then one of the prime elements is divisible by $p$ and hence equal to $p$, a contradiction since we chose elements of $\mathcal{H}$ to be primes $>k_{0}$. 
The main strategy of the proof goes back to the paper by Goldston, Pintz and Yildirim [GPY09] where they consider

$$
\begin{gathered}
S_{1}=\sum_{n \sim x} f(n), \\
S_{2}=\sum_{n \sim x}\left(\sum_{h \in \mathcal{H}} \theta(n+h)\right) f(n) .
\end{gathered}
$$

The idea is to show that for some admissible $\mathcal{H}$, we have

$$
S_{2}-(\log 3 x) S_{1}>0 \text {. }
$$

This would imply that there are at least two primes among the sequence

$$
n+h_{1}, \ldots, n+h_{k},
$$

for some $n \sim x$. They choose, $f(n)=\lambda(n)^{2}$ with

$$
\lambda(n)=\frac{1}{(k+\ell) !} \sum_{d \mid P(n), d<D} \mu(d) g(d),
$$

where

$$
g(d)=\left(\log \frac{D}{d}\right)^{k+\ell}
$$

where

$$
P(n)=\prod_{h \in \mathcal{H}}(n+h) .
$$

What is now needed is a good upper bound for $S_{1}$ and a good lower bound for $S_{2}$. This is the same strategy adopted in [GPY09]. To elaborate, let $C_{i}(d)$ be the set of solutions $(\bmod d)$ for $P\left(n-h_{i}\right) \equiv 0$ $(\bmod d)$ and define the singular series $\mathfrak{S}$ by

$$
\mathfrak{S}=\prod_{p}\left(1-\frac{\nu_{p}(\mathcal{H})}{p}\right)\left(1-\frac{1}{p}\right)^{-k} .
$$

With

$$
T_{1}^{*}=\frac{1}{(k+2 \ell) !}\left(\begin{array}{c}
2 \ell \\
\ell
\end{array}\right) \mathfrak{S}(\log D)^{k+2 \ell}+o\left((\log x)^{k+2 \ell}\right.
$$

and

$$
T_{2}^{*}=\frac{1}{(k+2 \ell+1) !}\left(\begin{array}{c}
2 \ell+2 \\
\ell+1
\end{array}\right) \mathfrak{S}(\log D)^{k+2 \ell+1}+o\left((\log x)^{k+2 \ell+1}\right),
$$

the argument of [GPY09] leads to

$$
S_{2}-(\log 3 x) S_{1}=\left(k T_{2}^{*}-(\log x) T_{1}^{*}\right) x+O\left(x(\log x)^{k+\ell}\right)+O(E)
$$

where

$$
E=\sum_{1 \leq i \leq k} \sum_{d<D^{2}} \mu(d) \tau_{3}(d) \tau_{k-1}(d) \sum_{c \in C_{i}(d)} \Delta(\theta ; d, c)
$$

and

$$
\Delta(\theta ; d, c)=\sum_{n \sim x, n \equiv c(\bmod d)} \theta(n)-\frac{1}{\phi(d)} \sum_{n \sim x} \theta(n) .
$$


Let us look at the main term. A quick calculation shows that it is

$$
\left(\frac{2 k(2 \ell+1)}{(k+2 \ell+1)(\ell+1)} \log D-\log x\right) \frac{(\log D)^{k+2 \ell}}{(k+2 \ell) !}\left(\begin{array}{c}
2 \ell \\
\ell
\end{array}\right) \mathfrak{S} x .
$$

We need to choose $D$ so that the term in brackets is positive. Let $D=x^{\alpha}$. The term in brackets is positive provided

$$
\frac{2 k(2 \ell+1)}{(k+2 \ell+1)(\ell+1)} \alpha-1>0 .
$$

That is, we need

$$
\alpha>\frac{(k+2 \ell+1)(\ell+1)}{2 k(2 \ell+1)}=\frac{1}{4}\left(1+\frac{2 \ell+1}{k}\right)\left(1+\frac{1}{2 \ell+1}\right) .
$$

From this, we see that if $k$ and $\ell$ are chosen sufficiently large and $\ell / k$ sufficiently small, the quantity on the right side is asymptotic to $1 / 4$. Thus, if we can choose $\alpha>1 / 4$ then we can find choices of $k$ and $\ell$ for which the main term is positive.

The error term is easily recognized to be related to the Bombieri-Vinogradov theorem. That theorem shows that for any $\alpha<1 / 4$, the error is negligible. So we seem to be at an impasse. However, a well-known conjecture of Elliott and Halbertsam [EH68] predicts that the error is negligible for any $\alpha<1 / 2$. This is where things stood in 2005 after the appearance of the paper [GPY09].

The new contribution of Zhang is that in the sums $T_{1}^{*}$ and $T_{2}^{*}$ (which are actually defined as terms involving the Möbius function and $g(d)$ ), he notes that terms with divisors $d$ having a large prime divisor are relatively small. So if we let $P$ be the product of primes less than a small power of $x$ and impose the condition that $d \mid P$ in the Bombieri-Vinogradov theorem then he is able to establish the following:

Theorem 2. For $1 \leq i \leq k$, we have

$$
\sum_{d<D^{2}, d \mid P} \sum_{P \in C_{i}(d)}|\Delta(\theta ; d, c)| \ll \frac{x}{\log ^{A} x},
$$

for any $A>0$ and $D=x^{\alpha}$ with $\alpha=1 / 4+1 / 1168$.

This theorem is the new innovation. Zhang admits that his choice of $k$ may not be optimal and that the optimal value of $k$ is "an open problem that will not be discussed in this paper." He was right because within days of his announcements, there was a flurry of internet activity to improve his numerical bounds. All of the developments are documented at:

michaelnielsen.org/polymath1/index.php?title=Bounded_ gaps_ between_ primes.

This led Maynard and Tao to develop an alternate method, first suggested by Selberg [Sel69] in his 1969 paper. We give a brief description of this in the next section.

\section{The Maynard-Tao method}

Instead of considering $\lambda(n)$ as above, the method of Maynard and Tao uses instead

$$
\sum_{d_{i} \mid n+h_{i}, 1 \leq i \leq k} \lambda_{d_{1}, \ldots, d_{k}} .
$$

In other words, we are sieving $n+h_{i}$ "independently" instead of combining all of them into one entity of $P(n)$. A further simplification of the analysis emerges if we set

$$
\lambda_{d_{1}, \ldots, d_{k}}=\mu\left(d_{1}\right) \cdots \mu\left(d_{k}\right) F\left(\frac{\log d_{1}}{\log D}, \ldots \frac{\log d_{k}}{\log D}\right)
$$


where $F$ is now a smooth function with compact support in $k$ variables. Such a weight was first suggested by Selberg as early as 1969, but seems to have not been developed by him. The idea is to express $F$ as a Fourier transform:

$$
F(t) e^{t}=\int_{\mathbb{R}^{k}} \eta(u) e^{-2 \pi i u t} d u .
$$

Here $t, u \in \mathbb{R}^{k}$ and $u t$ denotes the dot product. This clever device allows one to expand out the square in the original sums $S_{1}$ and $S_{2}$ above and then replace the function $F$ with the Fourier transform. Interchanging the summations, we obtain an integral whose integrand is a Dirichlet series whose behaviour is well-determined. One can derive an asymptotic formula relatively painlessly and thus obtain the final result involving now the parameter $k$ as well as the level of distribution $\theta$ appearing in the Bombieri-Vinogradov method.

More precisely, what they show is that there is a constant $M_{k}$ such that there are infinitely many integers $n$ such that at least $\left[\theta M_{k} / 2\right]$ of the $n+h_{i}$ are prime. The behaviour of $M_{k}$ is such that

$$
M_{k}>\log k+O(\log \log k) .
$$

This means that any positive level of distribution is sufficient to deduce bounded gaps between prime numbers.

The Maynard-Tao method also has other virtues. For example, one can prove that for any fixed natural number $m$,

$$
\liminf _{n}\left(p_{n+m}-p_{n}\right) \ll m^{3} e^{4 m} .
$$

Assuming the Elliott-Halberstam conjecture, that is, that the level of distribution in the BombieriVinogradov theorem is 1 , the gap can be reduced to 6 . These are tremendous results and signal a new era in sieve theory.

Acknowledgements. I would like to thank the referee for helpful remarks.

\section{References}

[BFI86] E. Bombieri, J. Friedlander and H. Iwaniec, Primes in arithmetic progressions to large moduli, Acta Math., 156(1986), 203-251.

[BFI87] E. Bombieri, J. Friedlander and H. Iwaniec, Primes in arithmetic progressions to large moduli II, Math. Annalen, 277 (1987), 361-393.

[BFI89] E. Bombieri, J. Friedlander and H. Iwaniec, Primes in arithmetic progressions to large moduli III, J. Amer. Math. Soc., 2(1989), 215-224.

[Dav00] H. Davenport, Multiplicative number theory, Springer, Third edition, 2000.

[Dus98] P. Dusart, Autour de la fonction qui compte le nombre de nombres prémiers, Ph.D. thesis, Université de Limoges, 1998.

[EH68] P. D. T. A. Elliott and H. Halberstam, A conjecture in number theory, Symp. Math., 4 (1968), 59-72.

[GPY09] D. Goldston, J. Pintz and C.Y. Yildirim, Primes in Tuples, I, Annals of Math., 170 (2009), 819-862.

[HL23] G. H. Hardy and J. E. Littlewood, Some problems of Partitio Numerorum III: on the expression of a number as a sum of primes, Acta Math., 44 (1923), 1-70.

[HW08] G. H. Hardy and E.M. Wright, An introduction to the theory of numbers, 6th Edition, Oxford University Press, 2008.

[May13] J. Maynard, Small gaps between primes, preprint, arXiv:1311.4600v2.

[Ram08] M. Ram Murty, Problems in analytic number theory, Springer, 2nd edition, 2008.

[Ram13a] M. Ram Murty, The twin prime problem and generalizations, Resonance, 18 (2013), $712-731$.

[Ram13b] M. Ram Murty, Ramanujan series for arithmetical functions, Hardy Ramanujan Journal, 36 (2013), $21-33$.

[Sel69] A. Selberg, Sieve Methods, Proc. Symp. Pure Math. ( SUNY, Stony Brook, N.Y. 1969), vol. XX, 311-351, Amer. Math. Soc., Providence, R. I., 1971.

[Tao13] T. Tao, Polymath8B: Variants of the Selberg sieve and bounded intervals containing many primes, available at: http://arxiv.org/pdf/1407.4897.pdf 
[Zha13] Y. Zhang, Bounded gaps between primes, Annals of Mathematics, 2013.

M. Ram Murty

Department of Mathematics

Queen's University

Kingston, Ontario, K7L 3N6

Canada

e-mail: murty@mast.queensu.ca 